\title{
EFFECT OF EMP FIELDS ON CELL MEMBRANE POTENTIALS
}

\author{
by Paul C. Gailey and Clay E. Easterly \\ Oak Ridge National Laboratory \\ P.O. Box 2008, Oak Ridge, TN 37831-6070
}

\begin{abstract}
A simple model is presented for cell membrane potentials induced during exposure to electromagnetic pulse (EMP). Usins calculated values of internal electric field strensth induced durins EMP exposure, the model predicts that cell membrane potentials of about $100 \mathrm{mV}$ may be induced for time frames on the order of $10 \mathrm{~ns}$. Possible biological effects of these potentials including electroporation are discussed.
\end{abstract}

\section{INTRODUCTION}

Electric field strengths occurring inside the body during EMP exposure are substantially higher than those produced during other types of field exposure. This concept is most easily understood by considering the body as a thick, lossy antenna with a broad resonance in the tens of megahertz range depending on height and grounding conditions. Near wholebody resonance, internal and external electric field strengths are comparable, and substantial energy may be absorbed by the body. This efficient energy absorption limits the field strengths to which an individual can be safely exposed near resonance for continuous wave or high-duty-cycle signals. Voluntary exposure guidelines in the United States recommend limiting power density to $1 \mathrm{~mW} / \mathrm{cm}^{2}$ near whole-bodyresonance frequencies. For a frec-space plane wave, this power density implies an electric field strength of about 60 $\mathrm{V} / \mathrm{m}$. Consequently, internal electric field strengths are seldom in excess of this value under ordinary conditions.

Electromagnetic pulse (EMP) exposures differ from typical environmental field exposures in that the body experiences very intense electromagnetic fields for a short time interval. A substantial fraction of the energy in the pulse may occur in the whole-body-resonance frequency range. During a 100 $\mathrm{kV} / \mathrm{m}$ EMP, electric field strengths inside the body can reach values as high as $200 \mathrm{kV} / \mathrm{m}$ for approximately $10 \mathrm{~ns}$ (Guy, 1990). These high field strengths produce no measurable heating of the body because very little energy is deposited during the short time interval. However, EMP-induced internal electric field strengths can be three or more orders of magnitude greater than those induced by the more common cw exposures, and questions about possible nonthermal biological effects cell are raised. In this paper, we discuss cell membrane potentials induced during EMP exposure.

\section{INTERNAL FIELD CHARACTERISTICS}

Guy (1990) predicted the currents and fields induced in a model of man using a method-of-moments computer code called NEC2D. Guy's model approximated the body as a series of cylindrical sections with varying electrical properties. Using an EMP with a peak electric field strength of 100 $\mathrm{kV} / \mathrm{m}$ and about $200 \mathrm{~ns}$ duration, he found that significant fields and currents were induced in the body for approximately 20 ns. As the induced current passes through the legs and into the ankles, it is confined to a decreasing cross-sectional area. This "focusing" of the current results in higher current density and internal electric fields. The effect is further enhanced by the low conductivity of bone resulting in confinement of most of the current in the higher conductivity regions of muscle and blood. Consequently, the highest exposures occur in the ankles where the peak current is predicted to reach 552 amperes and resulting current density is $1.75 \times 10^{5} \mathrm{~A} / \mathrm{m}^{2}$. The peak internal electric field associated with this current density is about $210 \mathrm{kV} / \mathrm{m}$ which is more than twice the value of the external electric field strength.

\section{SIMPLE MODEL FOR MEMBRANE POTENTIALS}

For the simplified model used here, the cell membrane is thought of as a lipid bilayer essentially two lipid molecules thick $(5-10 \mathrm{~nm})$. It is important to recognize that actual cell membranes are composed of a variety of different lipids, contain cholesterol within the molecular matrix, and are interspersed with various macromolectiles that serve as receptors, ion pumps, and other functions. Lipid bilayers exhibit very low conductivities and can be considered nonconducting to a first approximation. A value of $1 \mu \mathrm{F} / \mathrm{cm}^{2}$ is often used for the capacitance per unit area.

Several approaches can be used to find the membrane potential induced during an EMP, but the simplest is to use the current density and membrane capacitance. If the current density is assumed constant over some time interval, then the membrane potential at the end of that time interval is simply

$$
V_{m}=\frac{J \cdot \Delta t}{C_{m}}
$$

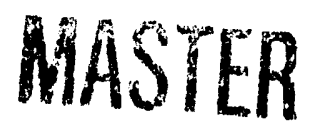


where

$$
\mathrm{J}=\text { current density, }
$$$$
\Delta t=\text { time interval, }
$$

and

$$
C_{m}=\text { capacitance por unit area. }
$$

This simple model assumes that the current flows uniformly through the membrane and extracellular medium surrounding the cell. This assumption is reasonable to a first approximation because the membrane impedance is small compared to the conductivity of the extracellular medium. However, if significant charging of the membrane occurs, the current will follow the gradient of the potential and tend to flow around the cell. The initial positive-going current pulse predicted by Guy can be approximated as a constant current density of 1.5 $x 10^{5} \mathrm{~A} / \mathrm{m}^{2}$ lasting for $10 \mathrm{~ns}$. Using these values, a maximum membrane potential of $150 \mathrm{mV}$ is predicted during the EMP exposure.

Considering a value of 0.2 microseconds for the charging time constant of the cell (Foster et. al., 1986), the effective duration of the EMP-induced current pulse is only about $5 \%$ or $10 \%$ of the charging time. It would appear on this basis that significant charging of the membrane will not take place, but one must also consider the limiting potential to which the membrane will charge in the infinite time limit. This limiting value is about 1.5 times the radius of the cell times the electric field strength in the surrounding medium. Using Guy's prediction of maximum field strength in the ankles $(210 \mathrm{kV} / \mathrm{m})$ and a cell radius of 10 microns, the membrane potential will reach about $3000 \mathrm{mV}$ in the infinite time limit or about $2000 \mathrm{mV}$ after one charging time constant. Considering that the EMP-induced charging lasts for about $5 \%$ of a time constant, the membrane potential will be roughly $5 \%$ of $2000 \mathrm{mV}$ or about $100 \mathrm{mV}$. Even though the maximum EMP-induced value is a small fraction of the fully-charged value, it still may represent a significant potential.

\section{BIOLOGICAL IMPLICATIONS}

While it is impossible to reliably draw conclusions from simple modeling results, it is reasonable to consider a range of possible effects. The cell membrane maintains a natural potential of about $70 \mathrm{mV}$ that is necessary for a variety of cell functions. Addition of the EMP-induced membrane potentials predicted here will result in the membrane potential being raised to about $170 \mathrm{mV}$ on one side of the cell, and depolarized to $-30 \mathrm{mV}$ on the other side of the cell. Depolarization of the membrane potential is generally unhealthy for a cell, but it is difficult to predict what effects, if any, will occur during 10 ns.

A potential biological effect that should be considered carefully is electroporation. Pore formation results in rapid changes in membrane potential and exposes the cell cytoplasm to the extracellular medium. Most studies of electroporation have used microsecond length pulses producing initial membrane potentials of over $1000 \mathrm{mV}$, but at least one study has examined the effects of $10 \mathrm{~ns}$ pulses (Benz et. al., 1980). This study revealed that 10 ns pulses producing initial membrane potentials near $1200 \mathrm{mV}$ caused reversible breakdowns of the membranes resulting in a sudden switch to a high conductance state. These results may be applicable to EMP exposures. Weaver et. al. (1992) have developed a numerical model of electroporation based on energy considerations which assumes that the resting conductance of lipid bilayers is due to very small pores which are spontaneously formed and destroyed. Application of pulsed fields below the values needed to produce electroporation provides energy for enlarging these natural pores, and may result in some biologically significant changes. Other potential effects include conformational changes and rotational forces on membrane macromolecules.

\section{ACKNOWLEDGEMENTS}

Research sponsored in part by Harry Diamond Laboratories' Inter-Agency Agreement No. 188-A066-A1 through Martin Marietta Energy Systems, Inc., under contract No. DEAC05-84OR21400 with the U.S. Department of Energy.

\section{REFERENCES}

Benz, R.; Zimmerman, U. Pulse-Length Dependence of the Electrical Breakdown in Lipid Bilayer Membrane, Biochemica et Biophysics Acta 57 (1980):637.

Foster, K.R.; Schwan, H.P. Dielectric permittivity and electrical conductivity of biological materials. In: Polk,C.; Postow, E.,eds. CRC handbook of biological effects of electromagnetic fields. Boca Raton, FL: CRC Press, 1986:27-96.

Guy, A.W. Analysis of time domain induced current, SAR, and absorbed energy distributions in human and nonhuman primates exposed to EMP electric fields. Final report to ERC Facilities service Corporation; Bioelectromagnetics Research Facility, University of Washington, Seattle, WA; 1990.

Weaver, J.C., Barnett, A. Progress Toward a Theoretical Model for Electroporation Mechanism: Membrane Electrical Behavior and Molecular Transport. In: Guide to Electroporation and Electrofusion, Academic Press, 1992. 

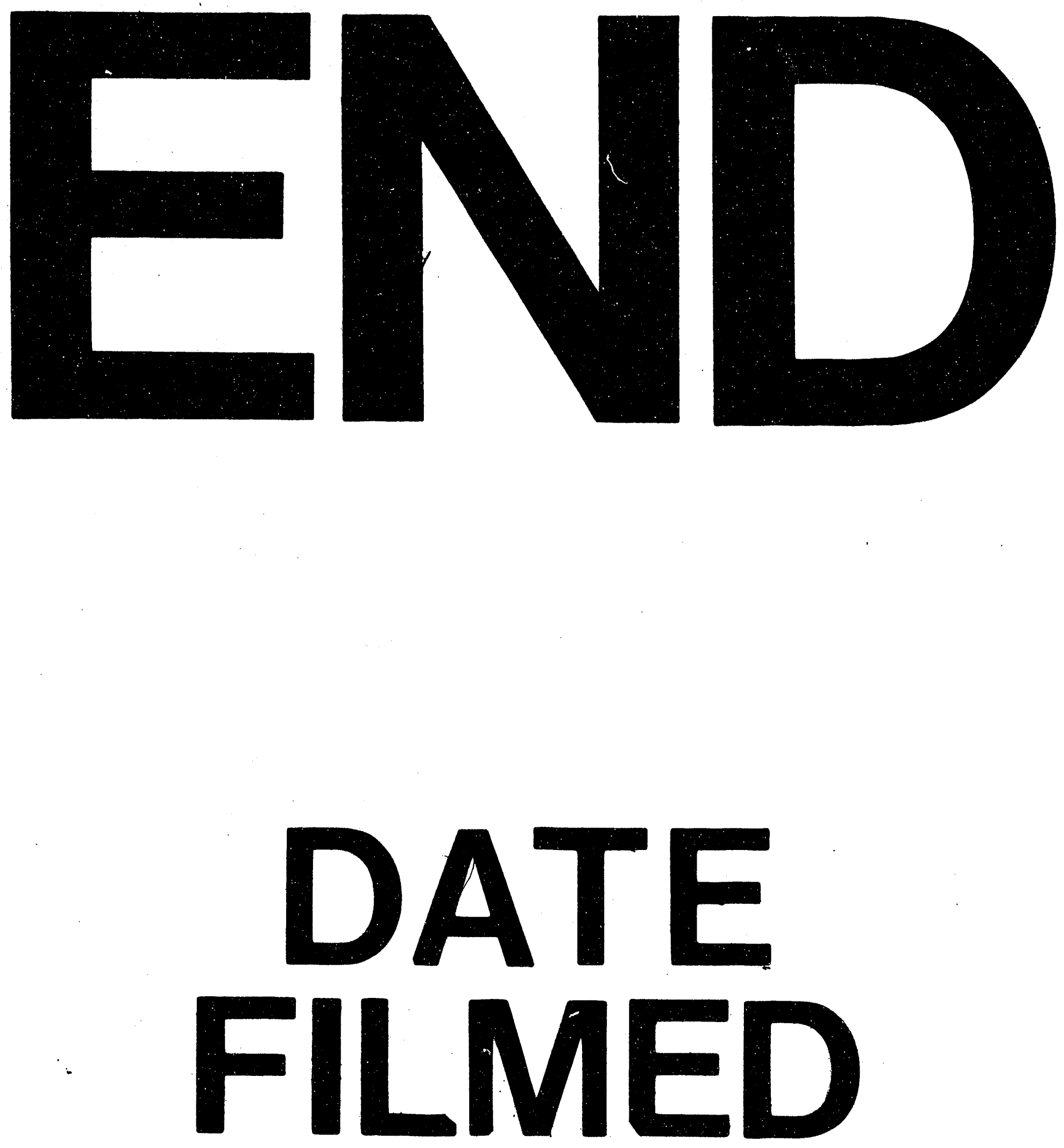

1

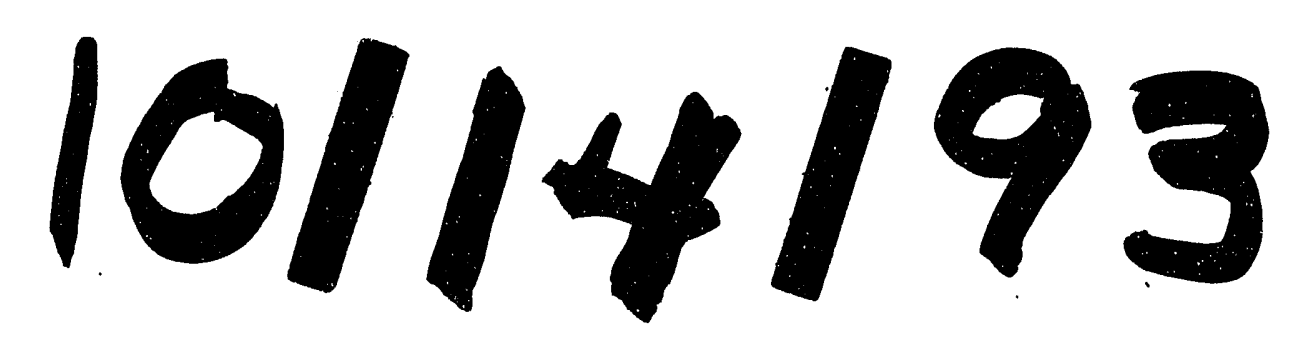


